\title{
A DISTRIBUTED, UNIVERSAL DEVICE FOR PLANAR PARTS FEEDING: UNIQUE PART ORIENTATION IN PROGRAMMABLE FORCE FIELDS
}

\author{
Karl F. Böhringer \\ Bruce R. Donald \\ Lydia E. Kavraki \\ Florent Lamiraux
}

\begin{abstract}
Programmable vector fields are an abstraction to represent a new class of devices for distributed, non-prehensile manipulation for applications in parts feeding, sorting, positioning, and assembly. Unlike robot grippers, conveyor belts, or vibratory bowl feeders, these devices generate force vector fields in which the parts move until they may reach a stable equilibrium pose.

Recent research in the theory of programmable vector fields has yielded open-loop strategies to uniquely position, orient, and sort parts. These strategies typically consist of several fields that have to be employed in sequence to achieve a desired final pose. The length of the sequence depends on the complexity of the part.

In this paper, we show that unique part poses can be achieved with just one field. First, we exhibit a single field that positions and orients any laminar part (with the exception of certain symmetric parts) into two stable equilibrium poses. Then we show that for any laminar part there exists a field in which the part reaches a unique stable equilibrium pose (again with the exception of symmetric parts). Besides giving an optimal upper bound for unique parts positioning and orientation, our work gives further evidence that programmable vector fields are a powerful tool for parts manipulation.

Our second result also leads to the design of "universal parts feeders," proving an earlier conjecture about their existence. We argue that universal parts feeders are relatively easy to build, and we report on extensive simulation results which indicate that these devices may work very well in practice. We believe that the results in this paper could be the basis for a new generation of efficient, open-loop, parallel parts feeders.
\end{abstract}




\section{INTRODUCTION}

Part manipulation is an important but also time-consuming operation in industrial automation. Parts and, in particular, small parts arrive at manufacturing sites in boxes and they need to be sorted and oriented before assembly. Traditionally part feeding and orienting has been performed with vibratory bowl feeders (Sandler, 1991, for example). These devices are customly designed for the orientation of a single part or a small number of parts and rely on mechanical filters to reject parts in unwanted orientations. Despite their widespread use, vibratory bowl feeders have several disadvantages: they have to be redesigned when the geometry of the part changes; they may damage parts that repeatedly run through the mechanical filters, etc.

Recent work investigates alternative ways for feeding parts in assembly workcells. Parts feeders that are programmed, rather than mechanically modified, offer an attractive solution since they can be used for a wide variety of parts (Goldberg, 1993; Akella et al., 1995; Erdmann, 1996; Böhringer et al., 1996b; Böhringer et al., 1999c). Practical considerations favor feeding methods that require little or no sensing, employ simple devices, and are as robust as possible (Erdmann and Mason, 1988; Goldberg, 1993; Canny and Goldberg, 1994; Akella et al., 1995; Böhringer et al., 1995; Böhringer et al., 1996b; Erdmann, 1996; Lynch, 1996; Wiegley et al., 1996). One of the proposed alternatives is the use of programmable vector fields (Böhringer et al., 1994; Coutinho and Will, 1997). The basic idea is the following: the field is realized on a planar surface on which the part is placed. The forces exerted on the contact surface of the part translate and rotate the part to an equilibrium configuration. The manipulation requires no sensing.

Until recently, work on force fields for manipulation has been dominated by the artificial potential fields pioneered by Khatib, Koditschek, and Brooks. While potential fields have been widely used in robot control (Khatib, 1986; Koditschek and Rimon, 1988; Rimon and Koditschek, 1992; Reif and Wang, 1995), micro-actuator arrays present us with the ability to explicitly program the applied force at every point in a vector field.

Current technology permits the implementation of certain vector fields in the microscale with actuator arrays built in micro electro mechanical system (MEMS) technology (Pister et al., 1990; Ataka et al., 1993; Fujita, 1993; Konishi and Fujita, 1994; Böhringer et al., 1994; Liu and Will, 1995; Cheung et al., 1997, for example), and in the macroscale with vibrating plates (Böhringer et al., 1995; Reznik and Canny, 1998). The 
flexibility and dexterity that programmable vector fields offer has led researchers to investigate the extent to which these fields can be useful. The work in (Böhringer et al., 1996b) analyzes the properties of vector fields that are suitable for sensorless manipulation and proposes novel manipulation strategies. These strategies typically consist of sequences of force vector fields that cascade the parts through multiple equilibria until a desired goal state is reached.

Programmable vector fields allow us to shift the complexity of parts feeding from the design of mechanical tracks, filters, and cut-outs to control algorithms and circuitry. No sensors or feeder re-design is required. However, the designs proposed in (Böhringer et al., 1996b) require control software, a clock, and, to some extent, synchronization between distributed actuators. In this paper we show that the device complexity can be further reduced. This work can be seen as an example of minimalist robotics (Canny and Goldberg, 1994; Böhringer et al., 1997a), which pursues the following agenda: For a given robot task, find the minimal configuration of resources required to solve the task. Minimalism is interesting because doing task $A$ without resource $B$ proves that $B$ is somehow inessential to the information structure of the task. ${ }^{1}$ This paper presents new results on minimalist part feeding, and gives optimal upper bounds on parts positioning and orienting.

Suppose we take the perspective of an architect seeking to simplify a parts feeder. MEMS arrays for programmable vector fields require control lines for programmability, plus a clock to switch between control strategies. In addition, control hardware and software are required, for example in a PC connected to the actuator array. Let us ask the minimalist question: Which components can be removed? This question devolves to a question about dynamical systems: Does there exist a single field in which every part $P$ has exactly one stable equilibrium (up to part symmetry)? It is somewhat remarkable that a purely architectural question can reduce to a conjecture about geometric dynamics.

This paper answers the above questions by presenting two specific device architectures. Assuming non-symmetric parts, the first design achieves exactly two stable equilibria without sensor feedback, clock, or control system. More precisely, unique positioning and orienting is reached modulo $180^{\circ}$ in orientation. The second design overcomes this limitation and for any non-symmetric part achieves unique positioning and orientation. We explain that our second result demonstrates the first known instance of a universal feeder/orienter (UFO) device (Böhringer et al., 1996b), i.e., a general purpose device that can uniquely position and orient any part without redesigning or reprogramming. 


\section{SQUEEZE FIELDS AND RADIAL FIELDS}

In this section we summarize some of the basic results in the theory of programmable vector fields that are necessary for the remainder of the paper. In a programmable force vector field, every point in the plane is associated with a force vector in the plane. For example, a unit squeeze field is defined as $\mathbf{f}(x, y)=-\operatorname{sign}(x)(1,0)$. When a part is placed into a squeeze field, it experiences a translation and re-orientation until a predictable equilibrium is reached. This property makes squeeze fields very useful for sensorless positioning and orienting strategies.

Given a polygonal part $P$ with $n$ vertices, it was shown in (Böhringer et al., 1994) that there exist $O\left(n^{2} k\right)$ stable equilibrium orientations for $P$ when placed in $\mathbf{f}$, where $k$ is the number of combinatorially distinct bisector placements for $P .{ }^{2}$ This result was used to generate strategies for unique parts posing (up to symmetry) by reducing the problem to a parts feeding algorithm developed by (Goldberg, 1993). The strategies have length $O\left(n^{2} k\right)$ and can be generated in $O\left(n^{4} k^{2}\right)$ time.

In (Böhringer et al., 1996b) this result was improved to plan lengths of $O(n k)$ and planning time $O\left(n^{2} k^{2}\right)$, by employing combined squeeze and unit radial fields. Unit radial fields are defined as $\mathbf{r}(x, y)=-\frac{1}{\sqrt{x^{2}+y^{2}}}(x, y)$ and are described in more detail in Section 5.

The original algorithm in (Böhringer et al., 1994) exhibited three key limitations:

1. While unique orientations could be achieved (modulo $180^{\circ}$ ) the final $(x, y)$ position was only known to lie somewhere along the last squeeze axis.

2. The dynamics of the part was assumed to be governed by quasistatic motion with separate phases of translation and rotation ("2PHASE assumption," see (Böhringer et al., 1994)).

3. Uniqueness of the final orientation was only possible modulo $180^{\circ}$ due to the inherent symmetry in the device design.

The improved algorithm in (Böhringer et al., 1996b) avoided limitations 1 and 2 , but item 3 remained. At the same time the improved algorithms required higher hardware complexity in the device design. In both approaches the part complexity $n$ appears in the upper bounds in the plan complexity, $O\left(n^{2} k\right)$ or $O(n k)$, respectively.

Using elliptic force fields $\mathbf{f}(x, y)=(-\alpha x,-\beta y)$ such that $0<\alpha<\beta$, this bound can be reduced to a constant number (2) independent of $n$ (Kavraki, 1997). We include this result in Section 4.

It was conjectured in (Böhringer et al., 1996b) that a field which combines a radial and gravitational field $\mathbf{r}+\delta \mathbf{g}$ (where $\mathbf{g}(x, y)=(0,-1)$ and 
Böhringer et al., Unique Part Orientation in Programmable Force Fields

\begin{tabular}{|c|c|c|c|c|c|}
\hline \multirow[t]{2}{*}{ Task } & \multicolumn{2}{|c|}{ Field(s) } & \multicolumn{3}{|c|}{ Complexity } \\
\hline & description & properties & planning & $\begin{array}{l}\text { plan } \\
\text { length }\end{array}$ & $\begin{array}{l}\text { \# goal } \\
\text { equil. }\end{array}$ \\
\hline translate & constant & $\begin{array}{l}\text { constant mag- } \\
\text { nitude and di- } \\
\text { rection }\end{array}$ & 0 & 1 & 0 \\
\hline position & $\begin{array}{l}\text { radial (Böhringer } \\
\text { et al., 1996b) }\end{array}$ & $\begin{array}{l}\text { constant } \\
\text { magnitude, } \\
\text { continuous } \\
\text { directions }\end{array}$ & 0 & 1 & $1 \quad(a)$ \\
\hline \multirow[t]{2}{*}{ orient } & $\begin{array}{l}\text { sequence of squeezes } \\
\text { (Böhringer et al., } \\
1994 \text { ) }\end{array}$ & $\begin{array}{l}\text { piecewise con- } \\
\text { stant magni- } \\
\text { tude and di- } \\
\text { rection }\end{array}$ & $O\left(k^{2} n^{4}\right)$ & $O\left(k n^{2}\right)$ & $2^{(b)}$ \\
\hline & $\begin{array}{l}\text { inertial (Kavraki, } \\
\text { 1997) }\end{array}$ & $\begin{array}{l}\text { smooth mag- } \\
\text { nitude, piece- } \\
\text { wise constant } \\
\text { direction }\end{array}$ & $O(1)^{(c)}$ & 1 & $2^{(b)}$ \\
\hline \multirow[t]{4}{*}{$\begin{array}{l}\text { position } \\
\text { and } \\
\text { orient }\end{array}$} & $\begin{array}{l}\text { sequence of orthog- } \\
\text { onal squeeze pairs } \\
\text { (Böhringer et al., } \\
\text { 1999a) }\end{array}$ & $\begin{array}{l}\text { piecewise con- } \\
\text { stant magni- } \\
\text { tude and di- } \\
\text { rection }\end{array}$ & $O\left(k^{2} n^{4}\right)$ & $O\left(k n^{2}\right)$ & $2 \quad(d)$ \\
\hline & $\begin{array}{l}\text { sequence of radial + } \\
\text { squeeze (Böhringer } \\
\text { et al., 1996b) }\end{array}$ & $\begin{array}{l}\text { piecewise con- } \\
\text { tinuous mag- } \\
\text { nitude and di- } \\
\text { rection }\end{array}$ & $O\left(k^{2} n^{2}\right)$ & $O(k n)$ & $2^{(d)}$ \\
\hline & elliptic & $\begin{array}{l}\text { smooth mag- } \\
\text { nitude and di- } \\
\text { rection }\end{array}$ & $O(1)^{(c)}$ & 1 & $2^{(d)}$ \\
\hline & radial-gravity & $\begin{array}{l}\text { smooth mag- } \\
\text { nitude and di- } \\
\text { rection }\end{array}$ & $O(1)^{(e)}$ & 1 & 1 \\
\hline
\end{tabular}

Table 1 Fields and algorithms for manipulation tasks with programmable force vector fields. The results on elliptic and radial-gravity fields are proven in this paper. Remarks:

(a) Translation equilibrium only, orientation is unconstrained.

(b) Orientation unique modulo $180^{\circ}$ symmetry, translation along squeeze line is unconstrained.

(c) Requires numerical computation of axes of inertia.

(d) Pose is unique modulo $180^{\circ}$ symmetry.

(e) Requires numerical computation of field parameter $\delta$. 
$\delta$ is a small positive constant), has the property of uniquely orienting and positioning parts. We call this field the radial-gravity field and we prove in Section 5 that for any non-symmetric part, there is a radial-gravity field inducing exactly one stable equilibrium. Our paper also includes a discussion on implementation issues relating to the radial-gravity field. Such a field could be used to build a universal parts feeder (inspired by the "universal gripper" as proposed by (Abell and Erdmann, 1996). ${ }^{3}$ ). In contrast to the universal manipulator fields proposed in (Reznik and Canny, 1998), such a device could uniquely position a part without the need of a clock, sensors, or programming.

Table 1 gives a summary of our results on part manipulation using programmable force vector fields. The first column of that table specifies a task. The three last columns show the complexity of generating a plan, the number of steps required during plan execution, and the number of final equilibria states for the particular task. The inertial field was defined as $\mathbf{f}(x, y)=-\operatorname{sign}(x)(x, 0)$. In Table $1, n$ denotes the number of vertices of the part and $k$ denotes the combinatorially distinct bisectors of the part.

\section{CONDITIONS FOR EQUILIBRIA}

In this section we give some definitions and establish the notation that will be used in the two following sections. We investigate the conditions for equilibrium for a part $w$ in the presence of a force field $\mathbf{f}: \mathbb{R}^{2} \rightarrow \mathbb{R}^{2}$. It is assumed that $w(x, y) \geq 0$, for $x, y \in \mathbb{R}$, and $W=\int_{\mathbb{R}^{2}} w(\mathbf{p}) d \mathbf{p}<\infty$. Here $w$ can be seen as the support (characteristic) function of the part, this function is 1 on the part and 0 elsewhere. We assume that the support of $w$ is compact.

Without loss of generality, the origin of the reference frame in the plane can be chosen as the center of mass of $w$ :

$$
\int_{\mathbb{R}^{2}} \mathbf{p} w(\mathbf{p}) d \mathbf{p}=\mathbf{0} .
$$

When the part is in configuration $q=(x, y, \theta)$, the resultant force is given by

$$
\mathbf{F}=\int_{\mathbb{R}^{2}} w(\mathbf{p}) \mathbf{f}\left(A_{\theta} \mathbf{p}+\mathbf{t}\right) d \mathbf{p}
$$

and the resultant torque at the center of mass is given by

$$
\mathbf{M}=\int_{\mathbb{R}^{2}} w(\mathbf{p})\left(A_{\theta} \mathbf{p}\right) \times \mathbf{f}\left(A_{\theta} \mathbf{p}+\mathbf{t}\right) d \mathbf{p},
$$

where $\mathbf{t}=(x, y)^{\top}$, and 
(a)
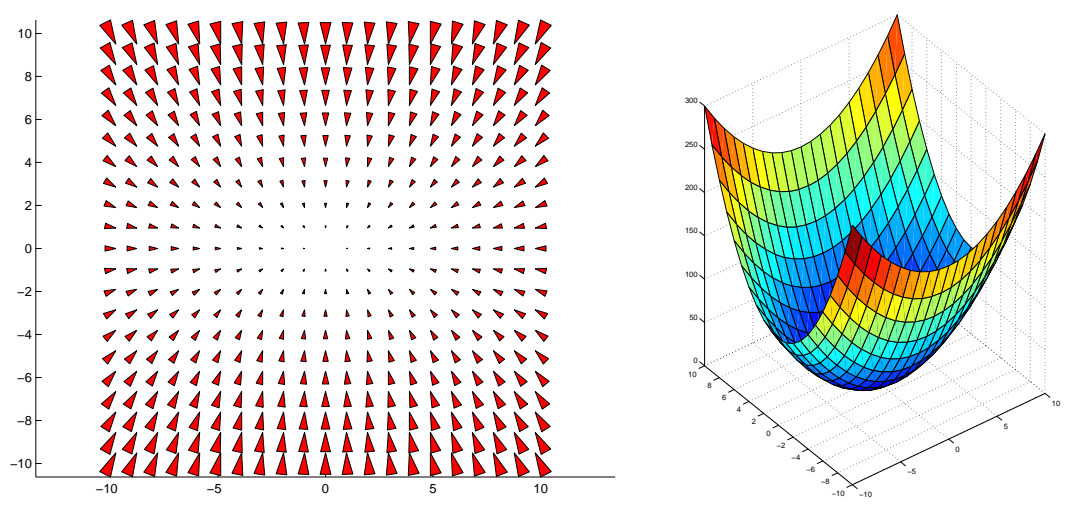

(b)

Figure 1 Elliptic force field (a) and corresponding potential field (b) for $\alpha=1$ and $\beta=2$.

$$
A_{\theta}=\left(\begin{array}{rr}
\cos \theta & -\sin \theta \\
\sin \theta & \cos \theta
\end{array}\right)
$$

is the rotation matrix of angle $\theta$. From now on, all integrals extend over $\mathbb{R}^{2}$ unless otherwise stated.

A total equilibrium is achieved when the resultant force and torque on the part is zero. For a total equilibrium the following two equations must hold:

$$
\begin{aligned}
\mathbf{F} & =\mathbf{0} \\
\mathbf{M} & =\mathbf{0} .
\end{aligned}
$$

\section{TWO STABLE EQUILIBRIUM ORIENTATIONS}

In this section we show a force field that can orient most parts into two stable equilibria. The field derives from an elliptic potential field and we will call it the elliptic field:

$$
\mathbf{f}(x, y)=(-\alpha x,-\beta y)
$$

where $\alpha$ and $\beta$ are two distinct positive constants. Without loss of generality let us assume that $\alpha<\beta$. Figure 1a displays one such force field with $\alpha=1$ and $\beta=2$. Note that this vector field is the negative gradient of the elliptic potential function $u(x, y)=\frac{\alpha}{2} x^{2}+\frac{\beta}{2} y^{2}$. This potential function is plotted in Figure $1 \mathrm{~b}$, for $\alpha=1$ and $\beta=2$. 


\subsection{FORCE AND MOMENT EQUILIBRIUM}

Force Equilibrium. We first establish the condition for the force equilibrium. If $(x, y)$ are the coordinates of the center of mass of $w$ in configuration $\mathbf{q}$ ( $W$ is defined in Section 3), the total force exerted on $w$, given by the left hand side of (1), is equal to

$$
(-\alpha W x,-\beta W y) \text {. }
$$

Condition (1) is thus equivalent to $(x, y)=(0,0)$. Therefore, in looking for equilibrium configurations $\mathbf{q}$, we only need to consider the configurations of the type $\mathbf{q}=(0,0, \theta)$.

Moment Equilibrium. We now proceed to the investigation of condition (2). It turns out that, for "most" parts $w$ and for whatever distinct positive values of $\alpha$ and $\beta$, there are exactly 4 values of $\theta$ for which (2) holds. This is shown below.

Taking into account the force equilibrium, the expression of the torque becomes now

$$
\mathbf{M}=\int w(\mathbf{p})\left(A_{\theta} \mathbf{p}\right) \times \mathbf{f}\left(A_{\theta} \mathbf{p}\right) d \mathbf{p} .
$$

The cross product of two vectors $\mathbf{v}_{1}=\left(x_{1}, y_{1}\right)$ and $\mathbf{v}_{2}=\left(x_{2}, y_{2}\right)$ is defined as $\mathbf{v}_{1} \times \mathbf{v}_{2}=\left|\begin{array}{ccc}\mathbf{i} & \mathbf{j} & \mathbf{k} \\ x_{1} & y_{1} & 0 \\ x_{2} & y_{2} & 0\end{array}\right|$ and the above equation gives after calculations

$$
\begin{aligned}
\mathbf{M}=(\alpha-\beta) & \left(\frac{\sin 2 \theta}{2} \int\left(x^{2}-y^{2}\right) w(x, y) d x d y\right) \cdot \mathbf{k}+ \\
(\alpha-\beta) & \left(\cos 2 \theta \int x y w(x, y) d x d y\right) \cdot \mathbf{k} .
\end{aligned}
$$

Thus, since $\alpha \neq \beta$, we have $\mathbf{M}=\mathbf{0}$ if and only if

$$
\frac{s_{20}-s_{02}}{2} \sin 2 \theta+s_{11} \cos 2 \theta=0 .
$$

In the above

$$
s_{m n}=s_{m n}(w)=\int_{\mathbb{R}^{2}} x^{m} y^{n} w(x, y) d x d y
$$

define moments of $w$. Equivalently, we want the vectors

$$
(\cos 2 \theta, \sin 2 \theta) \text { and }\left(s_{11}, \frac{1}{2}\left(s_{20}-s_{02}\right)\right)
$$

to be orthogonal. We now have to distinguish two cases. 
"SYMMETRY": $s_{11}=0$ and $s_{02}=s_{20}$.

Clearly in this case (5) is satisfied for all $\theta \in[0,2 \pi)$ and we have equilibrium regardless of orientation. When a part is in equilibrium for all $\theta$, we say that orientation fails for the part.

"ASYMMETRY": $s_{11} \neq 0$ or $s_{02} \neq s_{20}$.

When $\theta$ goes from 0 to $2 \pi$ the vector $(\cos 2 \theta, \sin 2 \theta)$ traverses the unit circle twice. The two vectors, $(\cos 2 \theta, \sin 2 \theta)$ and $\left(s_{11}, \frac{1}{2}\left(s_{20}-s_{02}\right)\right)$ will be orthogonal for exactly 4 values of $\theta$, say $\theta_{1}=\theta_{0}, \theta_{2}=\theta_{0}+\pi, \theta_{3}=\theta_{0}+\frac{\pi}{2}$, and $\theta_{4}=\theta_{0}+\frac{3 \pi}{2}$. In addition, either the first pair of them is stable and the second unstable, or vice versa. The reason is that the sign of $\mathbf{M}$ in (4) determines the direction in which moment $\mathbf{M}$ rotates the part. If this sign is positive, $\mathbf{M}$ rotates the part counter-clockwise, else the rotation is done clockwise (see also (Böhringer et al., 1996b)). While $(\cos 2 \theta, \sin 2 \theta)$ is rotated around the vector $\left(s_{11}, \frac{1}{2}\left(s_{20}-s_{02}\right)\right)$, the sign of the left hand side of (5) changes after the two vectors attain an orthogonal orientation. Hence, we observe sign changes of the left hand side of (5) for the 4 values of $\theta$ given above. Let $\theta_{1}$ and $\theta_{2}$ be the roots of (5) for which the sign of its left hand side changes from a negative value to a positive value while moving in a counter-clockwise direction. Since we assumed that $\alpha-\beta<0, \theta_{1}$ and $\theta_{2}$ indicate stable equilibrium configurations of the part (see equation 4 ), whereas $\theta_{3}$ and $\theta_{4}$ are unstable configurations.

This leads to the following theorem.

Theorem 1 Let $w: \mathbb{R}^{2} \rightarrow \mathbb{R}$ be a part with finite $s_{i j}$ with $i+j \leq 2$ and whose "center of mass" is at $\mathbf{0}$, and let $\mathbf{f}(x, y)=(-\alpha x,-\beta y)$, with $0<\alpha<\beta$, be the underlying force field.

"SYMmETRY": If $s_{11}=s_{20}-s_{02}=0$ the part $w\left(A_{\theta} \mathbf{p}+\mathbf{t}\right)$ is at (force and moment) equilibrium whenever $\mathbf{t}=\mathbf{0}$.

"ASYMMETRY": Otherwise, the distribution $w\left(A_{\theta} \mathbf{p}+\mathbf{t}\right)$ is in equilibrium only when $\mathbf{t}=0$ and for exactly 4 distinct values of $\theta \in[0,2 \pi)$. These 4 values of $\theta$ are $\frac{\pi}{2}$ apart and only 2 of them, say $\theta_{0}$ and $\theta_{0}+\pi$, represent stable equilibria, the others, $\theta_{0}+\frac{\pi}{2}$ and $\theta_{0}+\frac{3 \pi}{2}$ being unstable.

\subsection{PREDICTION OF EQUILIBRIA}

In practice, we seek to orient a finite part and it is very easy to compute with numerical techniques the values of $s_{11}, s_{20}$, and $s_{02}$. We can thus predict, for a given part, whether it will have 2 stable equilibria in the force field considered. The equilibrium orientations can be calculated using (5). Note that the equilibrium configurations of a part are independent of $\alpha$ and $\beta$, as long as $0<\alpha<\beta$.

Figure 2 shows the orientation of a polygonal part, called the ratchet, under the elliptic field with $\alpha=1$ and $\beta=2$. 


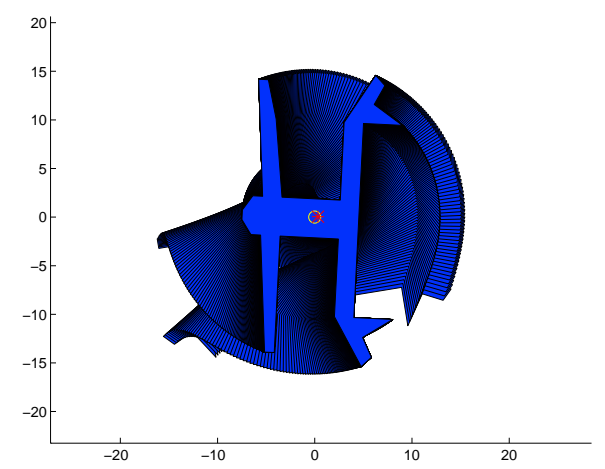

Figure 2 Orientation of a polygonal part under the elliptic force field with $\alpha=1$ and $\beta=2$.

In many cases it is clear that a part will have many equilibrium orientations. For example, consider a planar part that is a regular $n$-gon. This part will be at equilibrium when its "center of mass," as defined in Section 3, is at $\mathbf{0}$ no matter what its orientation is. The "center of mass" in this case is the centroid of its $n$-gon surface. Suppose now that the part had only two equilibria $\theta_{0}$ and $\theta_{0}+\pi$ and that the part is at equilibrium $\theta_{0}$. If we rotate the part by $\frac{2 \pi}{n}$ then we should have an equilibrium again, due to the geometrical symmetry of the part. Hence, since this part can not have only two equilibrium orientations it must be in equilibrium for any value of $\theta$, according to Theorem 1 . Indeed, for this part, it can be shown that $s_{11}=s_{20}-s_{02}=0$. Note that symmetry and asymmetry as in the above theorem do not always correspond to the notion of geometric symmetry and asymmetry, i.e. there may exist parts that are not geometrically symmetric but are symmetric according to the definitions above.

Equilibria, Principal Axes, and Part Symmetry. The constructive proof of Theorem 1 provides a method to predict the stable and unstable equilibria of any two-dimensional part $w$. For a given $w$ we determine its center of mass $\mathbf{c}$ and the angles $\theta_{1}, \ldots, \theta_{4} . w$ is in stable equilibrium in the force field $\mathbf{f}$ if and only if the line through $\mathbf{c}$ at angle $\theta_{1}$ coincides with the $x$-axis.

Readers familiar with theoretical mechanics will recognize the analogy between the proof of Theorem 1 and the transformation equations for moments and products of inertia. These equations are the basis for the argument that the principle axes of any two-dimensional part are 
perpendicular. It is worthwile to explore this analogy in more detail. For any part, there exists a coordinate frame such that $s_{11}=0$. The axes of this coordinate frame are the principal axes of inertia of the part (i.e., axes with maximum or minimum moment of inertia). It can be shown that these axes intersect at the center of mass c. From the previous computations, it is easy to deduce that in the two stable configurations, these axes are lined up with the axis of the force field. More specifically, $s_{20}$ and $s_{02}$ are the second area moments of $w$, often denoted $I_{x}$ and $I_{y}$, and $s_{11}=I_{x y}$ is the product of inertia. The line through $\mathbf{c}$ at angles $\theta_{1}$ or $\theta_{2}$ (corresponding to the stable equilibrium) is the major principal axis, and the line through $\mathbf{c}$ at angles $\theta_{3}$ or $\theta_{4}$ (corresponding to the unstable equilibrium) is the minor principal axis. These observations explain why the equilibrium is independent of the values of $\alpha$ and $\beta$ as long as $\alpha<\beta$.

Since all axes of symmetry are principal axes, it further follows that a sufficient condition for "SYMMETRY" as defined in Theorem 1 is that $w$ has two non-perpendicular axes of symmetry. Conversely, a necessary condition for "SYMMETRY" is that the product of inertia of $w$ must be zero for any axis through $\mathbf{c}$, and that the moment of inertia is equal for all axes through c. For more details on principal axes and moments of inertia, see for example (Meriam and Kraige, 1997).

\section{ONE STABLE EQUILIBRIUM ORIENTATION}

We now exhibit a class of force fields that induce one stable equilibrium for most parts. These fields are combinations of a unit radial and gravity field and we will call them radial-gravity fields:

- A unit radial field $\mathbf{r}$ is defined by: $\mathbf{r}(x, y)=-\frac{1}{\sqrt{x^{2}+y^{2}}}(x, y)$.

- A unit gravity field $\mathbf{g}$ is given by $\mathbf{g}(x, y)=(0,-1)$.

- For a given $\delta \in \mathbb{R}$, the radial-gravity field is defined as the sum of a unit radial field $\mathbf{r}$ and a gravity field $\mathbf{g}$ scaled by $\delta: \mathbf{f}_{\delta}=\mathbf{r}+\delta \mathbf{g}$.

Figure 3 plots a radial-gravity field for which $\delta=0.4$.

\subsection{FORCE AND MOMENT EQUILIBRIUM}

In this section we reason with potential fields instead of using directly equations (1) and (2). First we notice that $\mathbf{f}_{\delta}$ derives from the potential field $u_{\delta}(x, y)=\sqrt{x^{2}+y^{2}}-\delta y$ and we define the following potential field over the configuration space $\mathcal{C}$ of the part:

$$
U_{\delta}(q)=\int w(\mathbf{p}) u_{\delta}\left(A_{\theta} \mathbf{p}+\mathbf{t}\right) d \mathbf{p} .
$$


(a)
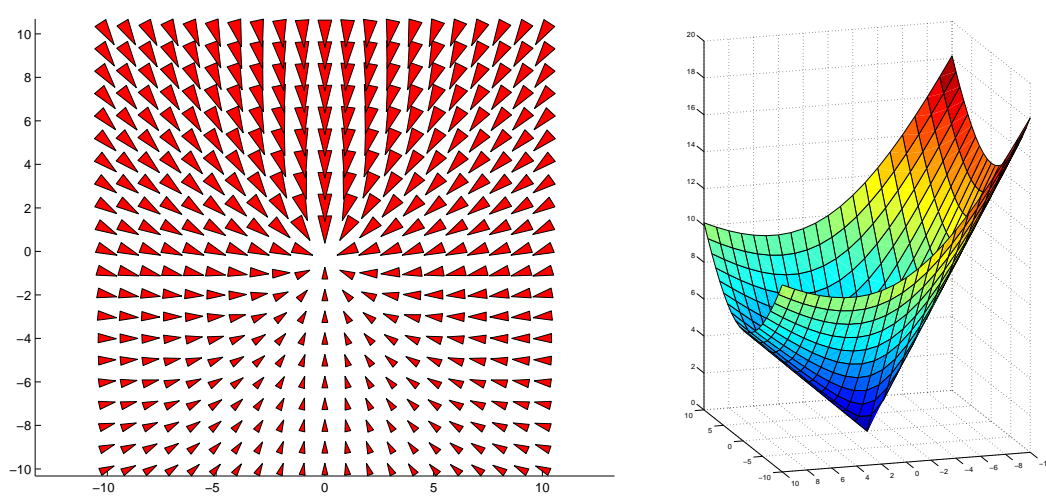

Figure 3 Radial-gravity field $\mathbf{f}_{\delta}=\mathbf{r}+\delta \mathbf{g}$ with $\delta=0.4$. (a) force field, (b) corresponding potential field.

A configuration $q$ is a stable equilibrium of the part if and only if $q$ is a local minimum of the function $U_{\delta}$.

In order to take advantage of the radial symmetry of $\mathbf{r}(x, y)$, we define a new system of coordinates $(X, Y, \theta)$ from the standard one by

$$
\begin{aligned}
X & =x \cos \theta+y \sin \theta \\
Y & =-x \sin \theta+y \cos \theta .
\end{aligned}
$$

The expression of $U_{\delta}$ in this new system of coordinates is obtained by a change of variable in the integral:

$$
\begin{aligned}
U_{\delta}(X, Y, \theta)= & \int w(\xi, \eta) \sqrt{(X+\xi)^{2}+(Y+\eta)^{2}} d \xi d \eta \\
& -\delta W(X \sin \theta+Y \cos \theta) .
\end{aligned}
$$

To establish the existence and uniqueness of a stable equilibrium, we proceed in two steps. First we state the existence and uniqueness of a local minimum of the potential field for any fixed $\theta$. This partial minimum is the force equilibrium. Then we study the curve of force equilibria when $\theta$ describes $\mathbf{S}^{1}$ and reason about moment equilibria. For our discussion below, we define the following functions:

$$
U_{\theta, \delta}(X, Y)=U(X, Y, \theta, \delta)=U_{\delta}(X, Y, \theta) .
$$

Force Equilibrium. A force equilibrium is a local minimum of $U_{\theta, \delta}$. Using common results of the theory of integration, we find that $U$ is of the class $C^{2}$ and that its partial derivatives with respect to $X$ and $Y$ are obtained by differentiating under the integral. The following proposition 
establishes the existence and uniqueness of a stable force equilibrium for a fixed $\delta$ by proving that the function $U_{\theta, \delta}$ is convex.

Proposition 2 If $\delta<1, U_{\theta, \delta}$ has a unique local minimum.

Proof: We first notice that for $\delta<1, U_{\theta, \delta}$ tends toward infinity with $(X, Y)$. We show then that $U_{\theta, \delta}$ is convex, i.e. the Hessian of $U_{\theta, \delta}$ is positive definite, that is its eigenvalues are both positive. This condition is fulfilled if and only if the trace and determinant of the Hessian are both positive:

$$
\begin{aligned}
& \operatorname{det} \text { Hess } U_{\theta, \delta}(X, Y)=\left|\begin{array}{cc}
\frac{\partial^{2} U_{\theta, \delta}}{\partial X^{2}}(X, Y) & \frac{\partial^{2} U_{\theta, \delta}}{\partial X \partial Y}(X, Y) \\
\frac{\partial^{2} U_{\theta, \delta}}{\partial X \partial Y}(X, Y) & \frac{\partial^{2} U_{\theta, \delta}}{\partial X}(X, Y)
\end{array}\right|>0 \\
& \operatorname{tr} \text { Hess } U_{\theta, \delta}(X, Y)=\frac{\partial^{2} U_{\theta, \delta}}{\partial X^{2}}(X, Y)+\frac{\partial^{2} U_{\theta, \delta}^{2}}{\partial Y^{2}}(X, Y)>0 .
\end{aligned}
$$

Let us compute the partial second derivatives of $U_{\theta, \delta}$ :

$$
\begin{aligned}
\frac{\partial^{2} U_{\theta, \delta}}{\partial X^{2}}(X, Y) & =\int w(\xi, \eta) \frac{(Y+\eta)^{2}}{\left((X+\xi)^{2}+(Y+\eta)^{2}\right)^{3 / 2}} d \xi d \eta \\
\frac{\partial^{2} U_{\theta, \delta}}{\partial Y^{2}}(X, Y) & =\int w(\xi, \eta) \frac{(X+\xi)^{2}}{\left((X+\xi)^{2}+(Y+\eta)^{2}\right)^{3 / 2}} d \xi d \eta \\
\frac{\partial^{2} U_{\theta, \delta}}{\partial X \partial Y}(X, Y) & =\int-w(\xi, \eta) \frac{(X+\xi)(Y+\eta)}{\left((X+\xi)^{2}+(Y+\eta)^{2}\right)^{3 / 2}} d \xi d \eta .
\end{aligned}
$$

From these expressions, we deduce easily that $\operatorname{tr} \operatorname{Hess} U_{\theta, \delta}(X, Y)>0$. Then using the identities $\left(\int f(\xi) d \xi\right)\left(\int g(\xi) d \xi\right)=\iint f(\xi) g(\eta) d \xi d \eta=$ $\iint f(\eta) g(\xi) d \xi d \eta$ we have:

$$
\begin{aligned}
& \operatorname{det} \operatorname{Hess} U_{\theta, \delta}(X, Y)=\frac{\partial^{2} U_{\theta, \delta}}{\partial X^{2}}(X, Y) \frac{\partial^{2} U_{\theta, \delta}}{\partial Y^{2}}(X, Y)-\left(\frac{\partial^{2} U_{\theta, \delta}}{\partial X \partial Y}(X, Y)\right)^{2} \\
& =\iint \frac{\left(Y+\eta_{1}\right)^{2}\left(X+\xi_{2}\right)^{2}-\left(X+\xi_{1}\right)\left(Y+\eta_{1}\right)\left(X+\xi_{2}\right)\left(Y+\eta_{2}\right)}{\left(\left(X+\xi_{1}\right)^{2}+\left(Y+\eta_{1}\right)^{2}\right)^{3 / 2}\left(\left(X+\xi_{2}\right)^{2}+\left(Y+\eta_{2}\right)^{2}\right)^{3 / 2}} d \xi_{1} d \eta_{1} d \xi_{2} d \eta_{2} \\
& =\frac{1}{2} \iint \frac{\left(Y+\eta_{1}\right)^{2}\left(X+\xi_{2}\right)^{2}+\left(Y+\eta_{2}\right)^{2}\left(X+\xi_{1}\right)^{2}}{\left(\left(X+\xi_{1}\right)^{2}+\left(Y+\eta_{1}\right)^{2}\right)^{3 / 2}\left(\left(X+\xi_{2}\right)^{2}+\left(Y+\eta_{2}\right)^{2}\right)^{3 / 2}} d \xi_{1} d \eta_{1} d \xi_{2} d \eta_{2} \\
& +\frac{1}{2} \iint \frac{-2\left(X+\xi_{1}\right)\left(Y+\eta_{1}\right)\left(X+\xi_{2}\right)\left(Y+\eta_{2}\right)}{\left(\left(X+\xi_{1}\right)^{2}+\left(Y+\eta_{1}\right)^{2}\right)^{3 / 2}\left(\left(X+\xi_{2}\right)^{2}+\left(Y+\eta_{2}\right)^{2}\right)^{3 / 2}} d \xi_{1} d \eta_{1} d \xi_{2} d \eta_{2} \\
& =\frac{1}{2} \iint \frac{\left(\left(Y+\eta_{1}\right)\left(X+\xi_{1}\right)-\left(Y+\eta_{2}\right)\left(X+\xi_{1}\right)\right)^{2}}{\left(\left(X+\xi_{1}\right)^{2}+\left(Y+\eta_{1}\right)^{2}\right)^{3 / 2}\left(\left(X+\xi_{2}\right)^{2}+\left(Y+\eta_{2}\right)^{2}\right)^{3 / 2}} d \xi_{1} d \eta_{1} d \xi_{2} d \eta_{2} \\
& >0 .
\end{aligned}
$$

where $w\left(\xi_{1}, \eta_{1}\right) w\left(\xi_{2}, \eta_{2}\right)$ has been omitted to make the notation clearer. 
Moment Equilibria. Having established the force equilibrium, we proceed to express it as a function of $\theta, \delta$.

Equilibrium curve. We denote by $\left(X^{*}(\theta, \delta), Y^{*}(\theta, \delta)\right)$ the unique force equilibrium relative to $\theta$ and by $\left(x^{*}(\theta, \delta), y^{*}(\theta, \delta)\right)$ its expression in the $(x, y, \theta)$ system of coordinates:

$$
\begin{aligned}
& x^{*}(\theta, \delta)=\cos \theta X^{*}(\theta, \delta)-\sin \theta Y^{*}(\theta, \delta) \\
& y^{*}(\theta, \delta)=\sin \theta X^{*}(\theta, \delta)+\cos \theta Y^{*}(\theta, \delta) .
\end{aligned}
$$

We call the curve of force equilibria $\left\{\left(x^{*}(\theta, \delta), y^{*}(\theta, \delta)\right), \theta \in \mathbf{S}^{1}\right\}$ equilibrium curve of parameter $\delta$.

When $\delta=0$ (pure radial field), due to the radial symmetry of the field, the set of equilibrium configurations is generated by the rotations of the part about one of its points called the pivot point (Böhringer et al., 1996b).

Proposition $3 X^{*}, Y^{*}, x^{*}, y^{*}$ are continuously differentiable.

Proof: The proof of this proposition is based on the implicit function theorem. Let us define the following function from $\mathbb{R}^{4}$ into $\mathbb{R}^{2}$

$$
F:(X, Y) \rightarrow\left(\begin{array}{c}
\frac{\partial U}{\partial X}(X, Y, \theta, \delta) \\
\frac{\partial U}{\partial Y}(X, Y, \theta, \delta)
\end{array}\right) .
$$

$\left(X^{*}, Y^{*}\right)$ minimizes the potential function $U_{\theta, \delta}$ for constant $\theta$ and $\delta$, and therefore fits the following implicit representation:

$$
F\left(X^{*}, Y^{*}, \theta, \delta\right)=0 .
$$

$F$ is continuously differentiable and the differential of the partial function $F_{\theta, \delta}$ of the variables $(X, Y)$ is exactly the Hessian of $U_{\theta, \delta}$. From Proposition 2 , this differential is invertible everywhere. All the hypotheses of the implicit function theorem are thus satisfied, and therefore $X^{*}(\theta, \delta)$ and $Y^{*}(\theta, \delta)$ are continuously differentiable. From relations (7) and (8), $x^{*}$ and $y^{*}$ are also continuously differentiable.

Let us now denote by $U_{\delta}^{*}(\theta)$ the minimum value of the potential function for each $\theta$. Then it is straightforward that $(X, Y, \theta)$ is a local minimum of $U_{\delta}$ if and only if $\theta$ is a local minimum of $U_{\delta}^{*}$ and $X=X^{*}(\theta, \delta)$ and $Y=Y^{*}(\theta, \delta)$. The following proposition establishes a relation between the derivative of $U_{\delta}^{*}$ and the position in the plane of the force equilibrium.

Proposition 4 For any $\theta \in \mathbf{S}^{1}$,

$$
\frac{d U_{\delta}^{*}}{d \theta}(\theta)=\delta W x^{*}(\theta, \delta)
$$


Proof: In this proof we omit $\delta$ in the expressions of $X^{*}$ and $Y^{*}$ to make the notation simpler. By definition $U_{\delta}^{*}(\theta)=U_{\delta}\left(X^{*}(\theta), Y^{*}(\theta), \theta\right)$. Differentiating this expression with respect to $\theta$ leads to

$$
\begin{aligned}
\frac{d U_{\delta}^{*}}{d \theta}(\theta)= & \frac{\partial U_{\delta}}{\partial X}\left(X^{*}(\theta), Y^{*}(\theta), \theta\right) \frac{d X^{*}}{d \theta}(\theta)+ \\
& \frac{\partial U_{\delta}}{\partial Y}\left(X^{*}(\theta), Y^{*}(\theta), \theta\right) \frac{d Y^{*}}{d \theta}(\theta)+ \\
& \frac{\partial U_{\delta}}{\partial \theta}\left(X^{*}(\theta), Y^{*}(\theta), \theta\right) \\
= & \frac{\partial U_{\delta}}{\partial \theta}\left(X^{*}(\theta), Y^{*}(\theta), \theta\right) \\
= & \delta W\left(\cos \theta X^{*}(\theta)-\sin \theta Y^{*}(\theta)\right) \\
= & \delta W x^{*}(\theta, \delta),
\end{aligned}
$$

since the partial derivatives of $U_{\delta}$ with respect to $X$ and $Y$ are null at $\left(X^{*}, Y^{*}\right)$.

Proposition 4 states that a stable equilibrium configuration corresponds to a value of $\theta$ where the equilibrium curve crosses the $\mathrm{y}$-axis from $x<0$ to $x>0$. Figure 7 (bottom left) shows the value of $U_{\delta}^{*}$ along the equilibrium curve for the ratchet part in the same figure and illustrates perfectly the linearity of the relation between $\frac{d U_{\delta}^{*}}{d \theta}$ and $x^{*}$. Indeed, it can be easily checked that the torque $\mathbf{M}$ is equal to the partial derivative of $U_{\delta}$ with respect to $\theta$.

Unique global equilibrium. We combine our results in propositions 2,3 , and 4 to establish the concluding theorem of this section.

Theorem 5 For any compact part $w$, if $\left(X^{*}(\theta, 0), Y^{*}(\theta, 0)\right) \neq(0,0)$ (i.e. the center of mass and the pivot point are distinct) then there exists $\delta>0$ such that $w$ has a unique stable equilibrium configuration under the potential field $U_{\delta}$.

Proof: First, let us notice that the curve $\left(X^{*}(\theta, 0), Y^{*}(\theta, 0)\right)$ is reduced to a point since when $\delta=0$, the potential field $U_{\delta}$ does not depend on $\theta$. Let us express this point in polar coordinates

$$
\begin{aligned}
X^{*}(\theta, 0) & =R \cos \varphi \\
Y^{*}(\theta, 0) & =R \sin \varphi .
\end{aligned}
$$

Then if $\left(X^{*}, Y^{*}\right) \neq(0,0)$, from relations (7) and (8), the curve $\left(x^{*}(\theta, 0), y^{*}(\theta, 0)\right)$ is a circle centered on $(0,0)$ (Figure 4$)$. We have

$$
\begin{aligned}
& x^{*}(\theta, 0)=R \cos (\theta+\varphi) \\
& y^{*}(\theta, 0)=R \sin (\theta+\varphi) .
\end{aligned}
$$




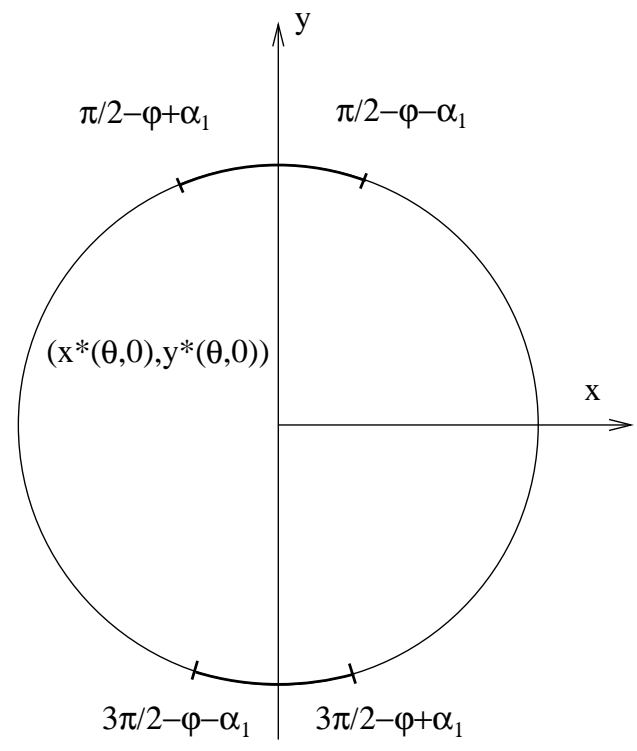

Figure 4 Decomposition of the equilibrium curve for $\delta=0$ into four intervals.

The current proof is based on the continuity of the functions $x^{*}$ and $y^{*}$ and their derivatives. We proceed in two steps: near $\pi / 2-\varphi$ and $3 \pi / 2-\varphi$, where $x^{*}(\theta, 0)$ crosses 0 , the variation of the tangent vector to the curve $\left(x^{*}(\theta, \delta), y^{*}(\theta, \delta)\right)$ can be made sufficiently small in order to prevent the curve to cross twice the y-axis. For the remaining values of $\theta$, the variation of the position of the curve can be bounded in such a way that the curve cannot cross the y-axis. The complete proof follows.

Let us recall that $\partial x^{*} / \partial \theta(\theta, \delta)$ is a continuous function and that $\partial x^{*} / \partial \theta(-\varphi+\pi / 2,0)=-R$ and $\partial x^{*} / \partial \theta(3 \pi / 2-\varphi, 0)=R$. Therefore there exists $\alpha_{1}>0$ and $\delta_{1}>0$ such that

$$
\begin{array}{ll}
\forall \delta<\delta_{1}, \forall \theta \in\left[-\varphi+\pi / 2-\alpha_{1},-\varphi+\pi / 2+\alpha_{1}\right], & \frac{\partial x^{*}}{\partial \theta}(\theta, \delta)<0 \\
\forall \delta<\delta_{1}, \forall \theta \in\left[-\varphi+3 \pi / 2-\alpha_{1},-\varphi+3 \pi / 2+\alpha_{1}\right], & \frac{\partial x^{*}}{\partial \theta}(\theta, \delta)>0 .
\end{array}
$$

These inequalities imply that the equilibrium curve does not cross more than once the y-axis on the corresponding intervals of $\theta$.

We are going now to show that for the remaining values of $\theta$, there exists a $\delta$ small enough such that the corresponding part of the equilibrium curve does not cross the y-axis. To make the notation clearer, let us define the following compact set

$$
I=\left[-\varphi+\pi / 2+\alpha_{1},-\varphi+3 \pi / 2-\alpha_{1}\right] \cup\left[-\varphi+3 \pi / 2+\alpha_{1},-\varphi+5 \pi / 2-\alpha_{1}\right] .
$$


Then for $\delta=0$ and $\theta \in I$, the equilibrium curve stays at a strictly positive distance from the $\mathrm{y}$-axis:

$$
\alpha_{2}=\operatorname{Inf}\left\{\left|x^{*}(\theta, 0)\right|, \theta \in I\right\}>0 .
$$

$x^{*}$ is continuous, thus its restriction to the compact set $I \times\left[0, \delta_{1}\right]$ is uniformly continuous. Therefore, there exists a constant $\delta_{2}>0$ such that

$$
\forall \theta \in I, \forall \delta \in\left[0, \delta_{2}\right],\left|x^{*}(\theta, \delta)-x^{*}(\theta, 0)\right|<\alpha_{2}
$$

and this condition ensures that the equilibrium curves does not cross the y-axis for $\theta \in I$ and $\delta<\delta_{2}$.

Therefore, for any $\delta<\min \left(\delta_{1}, \delta_{2}\right)$, the equilibrium curve crosses the $\mathrm{y}$-axis exactly twice. Once in each direction.

\subsection{PREDICTION OF EQUILIBRIA}

The previous computation shows that if a part has a pivot point different from the center of mass, then there exists a small value of $\delta$ to uniquely orient this part. However, this does not mean that there exists one unique value of $\delta$ orienting any part. In other words, the combination of a radial unit field and a gravitational field is a strategy that can orient almost any part, but for each part the maximum $\delta$ is different. For each part, the value of $\delta_{\max }$ can be computed numerically. These computations are discussed in more detail in Section 6.1.

Figure 5 shows equilibrium curves for the ratchet for different values of $\delta$. In this example, we can see that for large $\delta$, the equilibrium curve crosses the $y$-axis several times, and thus the minimum is not unique anymore. An annealing process may be used to determine $\delta$. The process starts with a value of $\delta$ just below 1 . This causes the part to be centered and oriented quickly. By reducing $\delta$ we ensure that eventually we obtain a field that uniquely orients the part.

Alternatively, we can determine the maximum value for $\delta$ for which the equilibrium is unique. By using numerical methods, we observe that for the ratchet for all $\delta$ values up to 0.46 the equilibrium is unique. This is demonstrated in Figure 5. Numerous simulation runs were performed to observe the behavior of the ratchet in the field $\mathbf{r}+0.46 \mathrm{~g}$. It consistently reaches the unique final position. Some of these simulation runs are shown in Figure 6. Figure 7 combines all these observations for the field $\mathbf{r}+0.3 \mathrm{~g}$. 
(a)
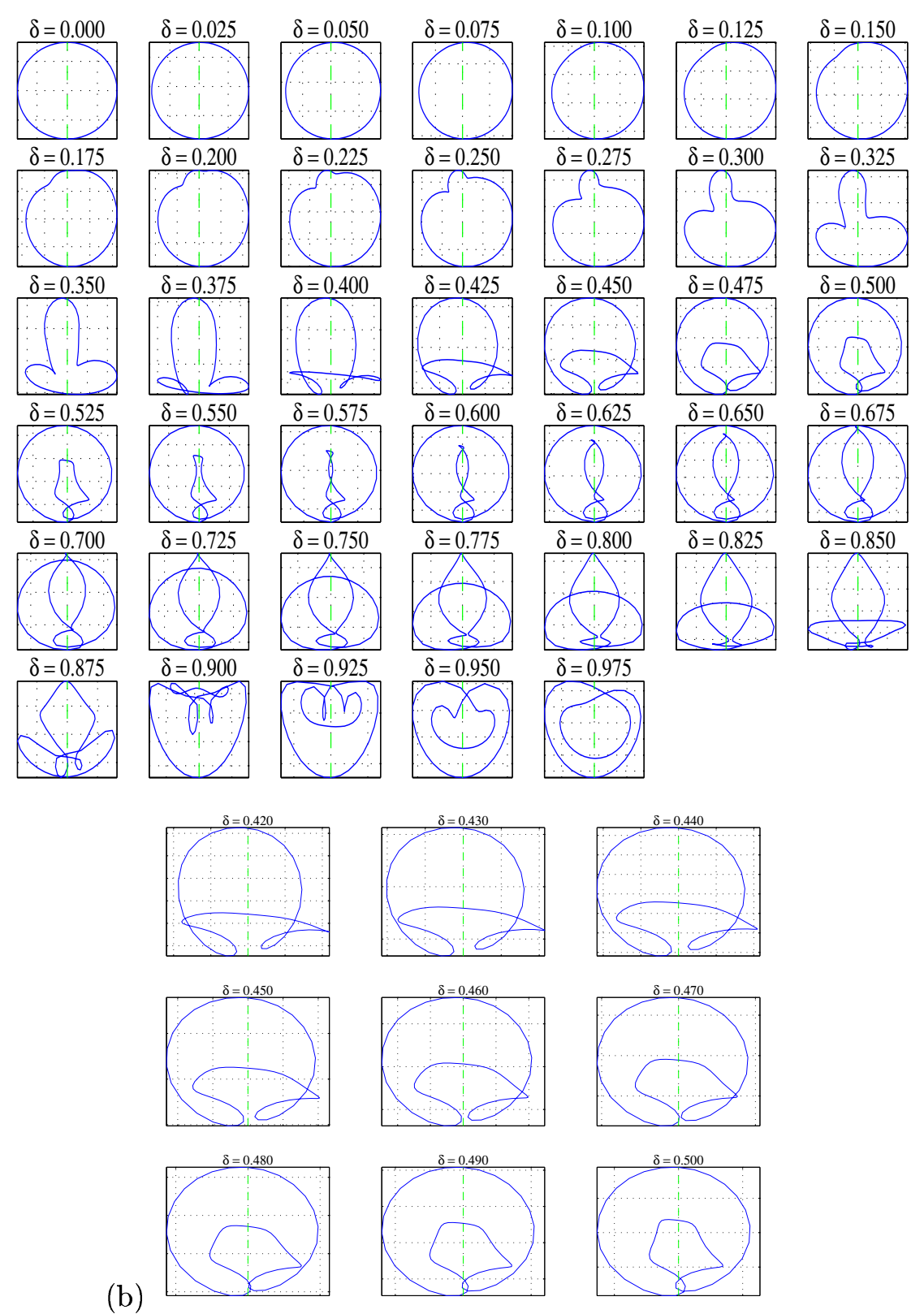

Figure 5 Detailed equilibrium curves for the ratchet: (a) Curves from $\delta=0$ to $\delta=0.975$, increment 0.025 . (b) Curves from $\delta=0.42$ to $\delta=0.50$, increment 0.01 . We observe that up to $\delta=0.46$ the curve has only two intersections with the $y$-axis, hence the equilibrium is unique. 

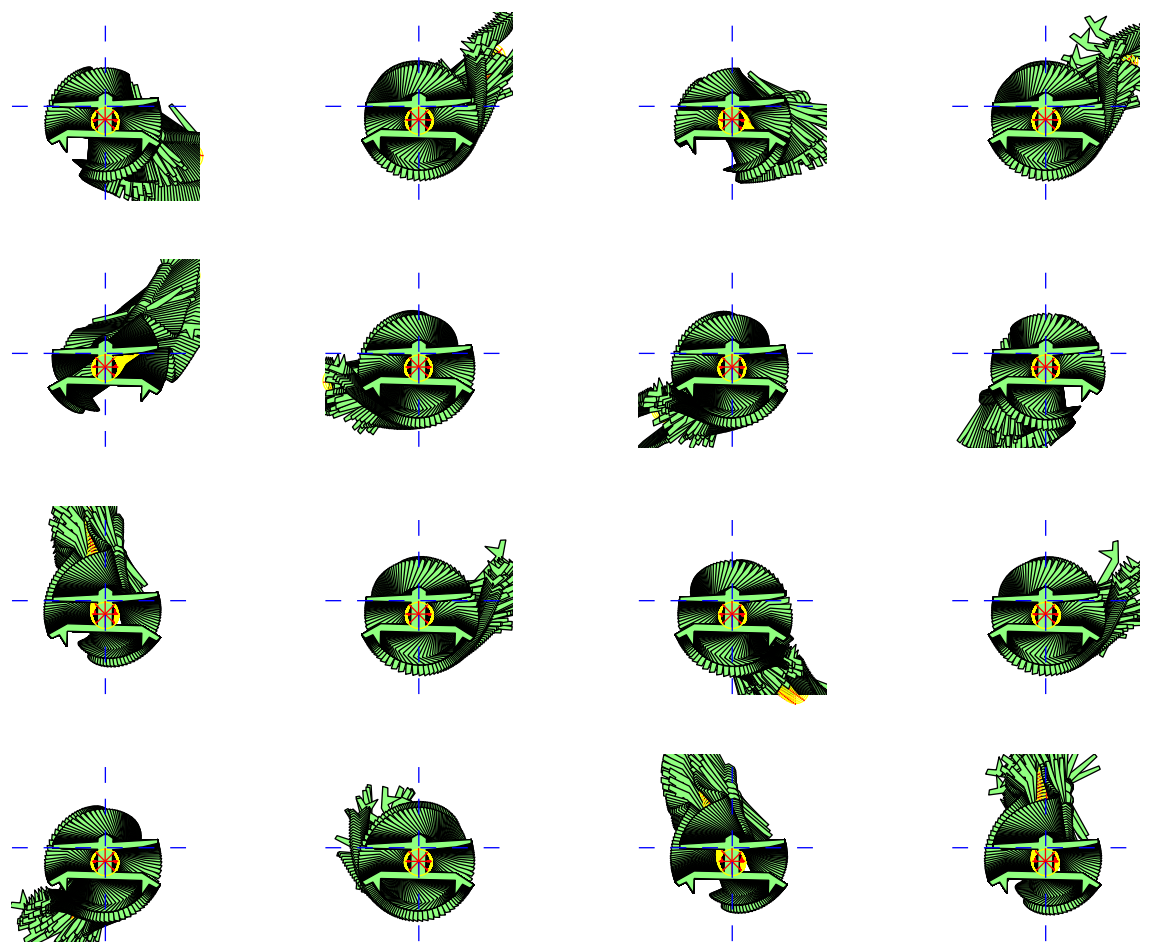

Figure 6 Simulation runs for the ratchet in the field $\mathbf{r}+0.46 \mathrm{~g}$. In all runs the part reaches the same final pose from a random initial pose.

\section{IMPLEMENTATION}

The previous sections show that there exist universal feeder/orienter devices that can uniquely position almost any part. We now briefly investigate practical issues on building such devices. To this end we pose two key questions:

- How difficult is it to build devices that implement programmable vector fields?

- How efficient is a universal feeder/orienter device in practice?

The first question concerns the initial setup cost as compared, e.g., with a vibratory bowl feeder or a robotic parts feeder. The second question addresses the issue that even though unique equilibria exist for almost all parts, it is not obvious a priori how quickly these equilibria will be reached. To obtain an answer to these questions we have built a comprehensive simulation and analysis system, and we have investigated 

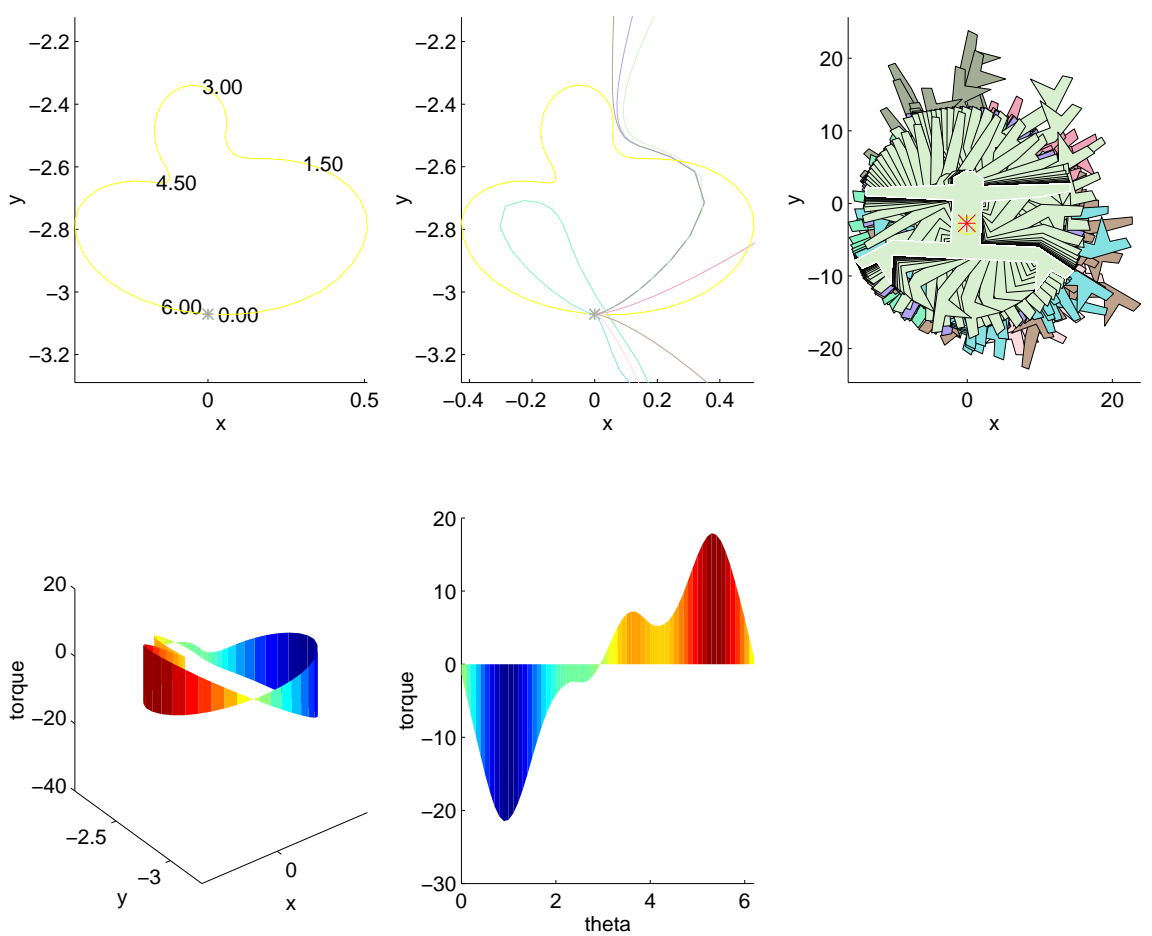

Figure 7 Analysis of the radial-gravity field $\mathbf{f}_{\delta}=\mathbf{r}+\delta \mathbf{g}$ with the ratchet and $\delta=0.3$. Top left: Equilibrium curve. Each point on this curve corresponds to a specific $\theta$ value, with $0 \leq \theta \leq 2 \pi$. Middle: Equilibrium curve with simulated trajectories of the ratchet. The center of mass always reaches the unique stable equilibrium (corresponding to the lower intersection of the curve with the $x$-axis). Right: Multiple simulation runs. The ratchet always reaches the same stable total equilibrium.

Bottom left: Equilibrium curve with corresponding torques. For a part whose center of mass is at $(x, y)$ in $\mathbf{f}_{\delta}$ the torque is directly proportional to $-x$. Middle: Torque as a function of part orientation $\theta$ when the part is in force equilibrium.

multiple designs that implement prototype devices for programmable vector fields.

\subsection{SIMULATION}

We have implemented a sophisticated simulator for programmable force vector fields in MATLAB. The system is capable of exact calculation of the force acting on polygonal parts in various fields, including squeeze, unit radial, gravity fields, and combinations thereof. To calculate the force acting on a polygon in the field, the polygon is triangulated 
and the force field is integrated over the individual areas. This can be done without numerical integration since there exist closed-form integrals for all these fields. To predict the part motion in the field, we have implemented a full dynamic simulator that includes inertia, viscous damping, and Coulomb friction. Figures 2, 6, and 7 show output of the dynamic simulator.

Force equilibria are determined numerically by solving the constraints $\mathbf{F}=0$ as given in equation (1). Equilibrium curves are determined numerically by calculating force equilibria for discrete part orientations. Figures 5 and 7-left/middle were generated in this way. Finally, pivot points are also determined numerically by solving Equation (1) for a part in a unit radial field.

Figures 6 and 7 consist of output from the software package and include dynamic simulation, numerical computation of force equilibria, and computation of torque when the part already is in force equilibrium (i.e., the torque associated with each point on the equilibrium curve). For the torque calculation see the last part of Figure 7.

\subsection{DEVICE CONSTRUCTION}

In Section 1 we have already mentioned some device designs that implement programmable vector fields. The idea of open-loop parts feeding is particularly attractive when dealing with very small or microfabricated parts, where precise feedback is difficult or extremely expensive. It also opens the opportunity for massively parallel positioning and assembly: since no control is required, the positioning process can be parallelized without communication overhead.

Towards this end, various researchers have demonstrated microfabricated actuator arrays based on MEMS (micro electro mechanical system) technology. These devices consist of a surface with potentially thousands or even millions of microscopic actuators, each of them capable of generating a unit force in a specific direction (Pister et al., 1990; Ataka et al., 1993; Fujita, 1993; Böhringer et al., 1994; Liu and Will, 1995, for example).

While MEMS actuator arrays may be useful to implement force fields that require high spatial resolution, alternative (macroscopic) designs are possible as well. In the following subsections we give some specific design ideas.

Elliptic Fields. The realization of elliptic fields could be achieved with MEMS actuator arrays (Böhringer et al., 1996a; Böhringer et al., 1997b), or arrays of motors (Luntz et al., 1997), and possibly with vibrating plates (Böhringer et al., 1995). The main challenge for vibrat- 


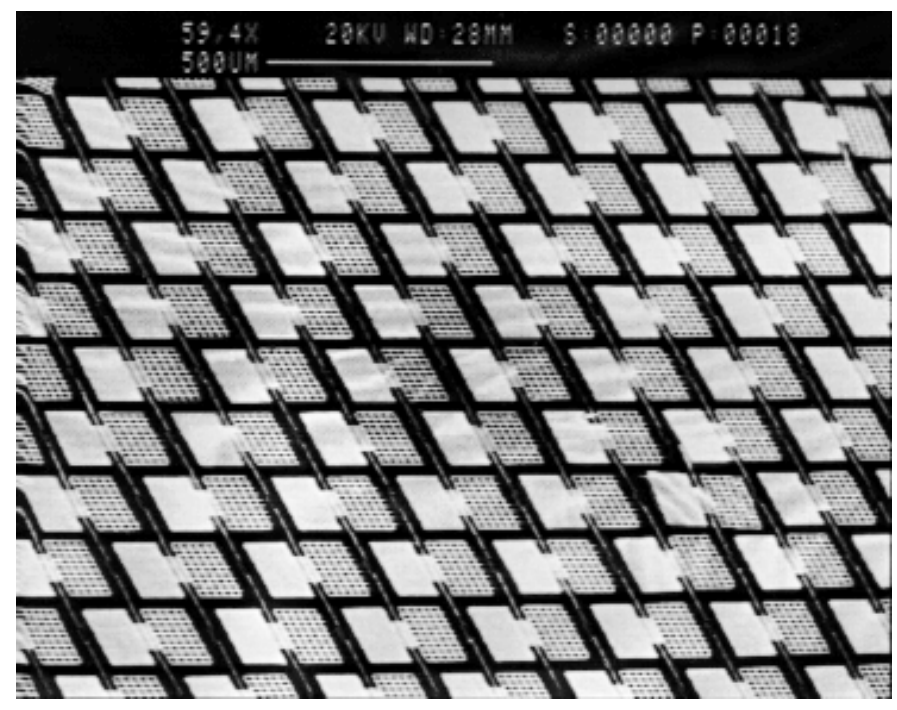

Figure 8 Unidirectional MEMS actuator array build on a silicon wafer. Each actuator is about $0.2 \mathrm{~mm}$ in size.

ing plates will be to obtain a surface that approximates the elliptic force profile with sufficient spatial resolution. Microscopic (MEMS) or macroscopic (motor) actuator arrays offer alternatives. Note that individual control of the actuators is not necessary; control by rows and columns only is sufficient. Furthermore, the proposed vector field could be implemented with a technology that allows the specification of a force only in one of the $x$ or $y$ directions at each pixel/actuator. Then two arrays, one controlled only in the $x$ direction and the other controlled only in the $y$ direction can be "interleaved." If the arrays are dense, the resulting force will be a force with the desired magnitude and direction. The main challenge for micro actuators remains the generation and control of forces over a sufficiently large range of force magnitudes.

Universal Fields. A prototype unidirectional array was built by (Böhringer et al., 1996a) (see Figure 8). This array can generate a unit gravity field. Its design could be modified such that the actuators are arranged in a circular pattern, which would result in a unit radial field. The variable gravity field could then be added simply by tilting the array accordingly (see Figure 9). Hence such a device would be relatively easy to build. The key observation is that with current MEMS technology it is easy to build actuator arrays with high spatial resolution $(\ll 1 \mathrm{~mm})$ and constant force, but it is difficult to build actuators with 


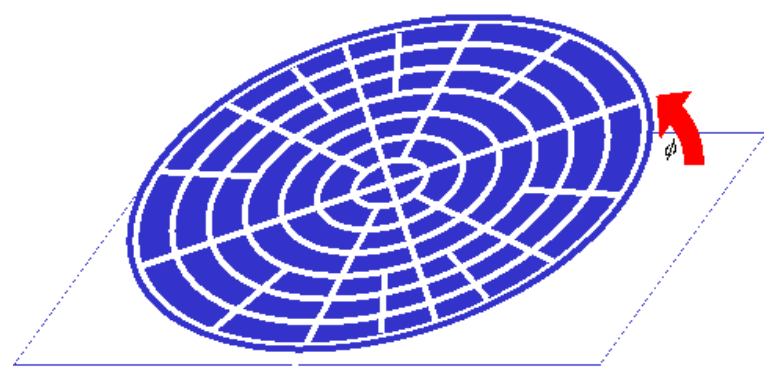

Figure 9 Conceptual design of an actuator array that implements a combined radialgravity field. Individual actuators are tiled in a circular array pattern. The array is tilted by an angle $\phi$ to add a gravity component $\delta \mathrm{g}$. Under some simplifying assumptions $\delta=\tan \phi$.

variable force. In addition, MEMS actuators can be easily arranged into arbitrary patterns (in particular, a radial pattern). Hence it is easy to build arrays that implement unit radial fields.

Alternatively, a resonating speaker, or a vibrating disk-shaped plate that is fixed at the center, might be used to create a radial force field.

\section{$7 \quad$ SUMMARY}

This paper proves the existence of devices for parts positioning and orienting that can bring arbitrary (non-symmetric) parts into exactly one or two stable equilibria. These devices are extremely simple: they do not require a feedback control, a clock, synchronization, or programming. Their functioning principle is based on force vector fields. Such a device could revolutionize industrial and precision parts handling.

This result opens the door for a multitude of new questions, some of which are briefly outlined below.

\section{Open Questions}

Parallelism. So far we have considered only the equilibria of one part in a force field. But what happens if two parts are placed into the field simultaneously? It is conceivable that the parts will settle in predictable configurations. This effect could be exploited for automated assembly.

When parts are initially placed far enough apart, it may be possible to implement several radial-gravity fields next to each other to achieve 
parallel positioning. This issue is particularly interesting since there is no overhead for parallelism in such a device, as no communication and control is required.

Symmetric parts. In Section 4 we have shown that elliptic fields achieve two equilibria for any part with $s_{11} \neq 0$ and $s_{20} \neq s_{02}$. Parts that do not satisfy this condition will be in neutral orientation equilibrium once their centers of mass reach the center of the elliptic field. Since the above conditions are not met for parts with rotational symmetry, these parts cannot be uniquely oriented in an elliptic field.

Similarly, Theorem 5 requires that the pivot point and center of mass of a part do not coincide. Thus, this result does not apply to rotationally symmetric parts such as, e.g., squares or hexagons. However, simulation results indicate that symmetric parts may still reach a unique equilibrium up to part symmetry. In case a part is symmetric, the user may not care about multiple equilibria as long as there exists no noticeable difference in the final poses. Therefore we generalize Theorem 5 to obtain the following conjecture: A radial-gravity field uniquely poses any part up to part symmetry.

Large $\delta$ values. We have shown that there always exists a $\delta_{\max }$ such that for all $0<\delta<\delta_{\max }$ we obtain a unique equilibrium. Figure 5 shows that for $\delta>\delta_{\max }$ the equilibrium curve becomes more complicated, causing multiple equilibria. However, as $\delta$ approaches 1 the curve becomes simpler again. Since higher $\delta$ values imply faster convergence, it would be interesting to know whether unique equilibria can be found for $\delta$ close to 1 .

\section{Acknowledgments}

Work on this paper by Karl Böhringer and Bruce Randall Donald has been supported in part by the National Science Foundation under Grant Nos. IRI-8802390, IRI9000532, IRI-9201699, IRI-9530785, IRI-9896020, NSF 9802068, NSF CDA-9726389, NSF EIA-9818299, NSF CISE/CDA-9805548, IIS-9906790, EIA-9901407, by a Presidential Young Investigator award to Bruce Donald, by an NSF/ARPA Small Grant for Exploratory Research No. IRI-9403903, by an NSF CISE Postdoctoral Associateship No. CDA-9705022 and an NSF CAREER award No. ECS-9875367 to Karl Böhringer, and in part by the Air Force Office of Sponsored Research, the Mathematical Sciences Institute, Intel Corporation, and AT\&T Bell laboratories. Work on this paper by Lydia Kavraki and Florent Lamiraux has been supported in part by NSF IRI-970228 and NSF CISE SA1728-21122N.

The authors would like to thank Eric Babson, Mike Erdmann, and Jonathan Luntz for valuable discussions and comments. 
Böhringer et al., Unique Part Orientation in Programmable Force Fields

\section{Notes}

1. In robotics, minimalism has become increasingly influential. (Raibert et al., 1993) showed that walking and running machines could be built without static stability. (Erdmann and Mason, 1996) showed how to do dextrous manipulation without sensing. (McGeer, 1990) built a biped, kneed walker without sensors, computers, or actuators. (Canny and Goldberg, 1994) argue that minimalism has a long tradition in industrial manufacturing, and developed geometric algorithms for orienting parts using simple grippers and accurate, low cost light beams. (Brooks, 1986) developed online algorithms that rely less extensively on planning and world models. (Donald et al., 1995; Böhringer et al., 1997a) built distributed teams of mobile robots that cooperate in manipulation without explicit communication.

2. For details on combinatorially distinct bisector placements see (Böhringer et al., 1999b).

3. In a universal gripper a part is free to rotate after being picked up from an arbitrary initial state. Its center of mass will settle at the unique minimum of potential energy, causing the part to reach a unique, predictable equilibrium.

\section{References}

Abell, T. L. and Erdmann, M. (1996). A universal parts feeder. Personal communication.

Akella, S., Huang, W. H., Lynch, K. M., and Mason, M. T. (1995). Planar manipulation on a conveyor by a one joint robot with and without sensing. In International Symposium of Robotics Research (ISRR).

Ataka, M., Omodaka, A., and Fujita, H. (1993). A biomimetic micro motion system. In Transducers - Digest Int. Conf. on Solid-State Sensors and Actuators, pages 38-41, Pacifico, Yokohama, Japan.

Böhringer, K.-F., Bhatt, V., Donald, B. R., and Goldberg, K. Y. (1999a). Sensorless manipulation using transverse vibrations of a plate. Algorithmica. Special Issue on Algorithmic Foundations of Robotics. Forthcoming.

Böhringer, K.-F., Bhatt, V., and Goldberg, K. Y. (1995). Sensorless manipulation using transverse vibrations of a plate. In Proc. IEEE Int. Conf. on Robotics and Automation (ICRA), pages 1989-1996, Nagoya, Japan.

Böhringer, K.-F., Brown, R. G., Donald, B. R., Jennings, J. S., and Rus, D. (1997a). Distributed robotic manipulation: Experiments in minimalism. In O. Khatib et al., editor, Experimental Robotics IV, Lecture Notes in Control and Information Sciences 223, pages 11-25. Springer Verlag, Berlin.

Böhringer, K.-F., Donald, B. R., and Halperin, D. (1999b). On the area bisectors of a polygon. Discrete and Computational Geometry. Forthcoming.

Böhringer, K.-F., Donald, B. R., and MacDonald, N. C. (1996a). Singlecrystal silicon actuator arrays for micro manipulation tasks. In Proc. 
IEEE Workshop on Micro Electro Mechanical Systems (MEMS), pages 7-12, San Diego, CA.

Böhringer, K.-F., Donald, B. R., and MacDonald, N. C. (1996b). Upper and lower bounds for programmable vector fields with applications to MEMS and vibratory plate parts feeders. In International Workshop on Algorithmic Foundations of Robotics (WAFR), Toulouse, France. Appeared in Algorithms for Robotic Motion and Manipulation, JeanPaul Laumond and Mark Overmars (Eds), pages 255-276, A. K. Peters, Ltd, 1997.

Böhringer, K.-F., Donald, B. R., and MacDonald, N. C. (1999c). Programmable vector fields for distributed manipulation, with applications to MEMS actuator arrays and vibratory parts feeders. Int. Journal of Robotics Research, 18(2):168-200.

Böhringer, K.-F., Donald, B. R., MacDonald, N. C., Kovacs, G. T. A., and Suh, J. W. (1997b). Computational methods for design and control of MEMS micromanipulator arrays. IEEE Computer Science and Engineering, pages 17-29.

Böhringer, K.-F., Donald, B. R., Mihailovich, R., and MacDonald, N. C. (1994). Sensorless manipulation using massively parallel microfabricated actuator arrays. In Proc. IEEE Int. Conf. on Robotics and Automation (ICRA), pages 826-833, San Diego, CA.

Brooks, R. (1986). A layered intelligent control system for a mobile robot. IEEE Journal of Robotics and Automation, RA(2).

Canny, J. and Goldberg, K. (1994). "RISC" for industrial robotics: Recent results and open problems. In Proc. IEEE Int. Conf. on Robotics and Automation (ICRA). IEEE.

Cheung, P., Berlin, A., Biegelsen, D., and Jackson, W. (1997). Batch fabrication of pneumatic valve arrays by combining mems with printed circuit board technology. In Proc. Symposium on Micro-Mechanical Systems, ASME International Mechanical Engineering Congress and Exhibition, pages 16-21, Dallas, TX.

Coutinho, M. and Will, P. (1997). The intelligent motion surface: a hardware/software tool for the assembly of meso-scale devices. In Proc. IEEE Int. Conf. on Robotics and Automation (ICRA), Albuquerque, New Mexico.

Donald, B. R., Jennings, J., and Rus, D. (1995). Information invariants for distributed manipulation. In Goldberg, K., Halperin, D., Latombe, J.-C., and Wilson, R., editors, Algorithmic Foundations of Robotics (WAFR), pages 431-459. A. K. Peters, Ltd, Wellesley, MA.

Erdmann, M. (1996). An exploration of nonprehensile two-palm manipulation: Planning and execution. In Giralt, G. and Hirzinger, G., editors, Robotics Research, pages 16-27. Springer Verlag. 
Erdmann, M. A. and Mason, M. T. (1988). An exploration of sensorless manipulation. IEEE Journal of Robotics and Automation, 4(4).

Erdmann, M. A. and Mason, M. T. (1996). Nonprehensile manipulation. In Robotic Motion and Manipulation, Toulouse, France.

Fujita, H. (1993). Group work of microactuators. In International Advanced Robot Program Workshop on Micromachine Technologies and Systems, pages 24-31, Tokyo, Japan.

Goldberg, K. Y. (1993). Orienting polygonal parts without sensing. Algorithmica, 10(2/3/4):201-225.

Kavraki, L. (1997). Part orientation with programmable vector fields: Two stable equilibria for most parts. In Proc. IEEE Int. Conf. on Robotics and Automation (ICRA), Albuquerque, New Mexico.

Khatib, O. (1986). Real time obstacle avoidance for manipulators and mobile robots. Int. Journal of Robotics Research, 5(1):90-99.

Koditschek, D. E. and Rimon, E. (1988). Robot navigation functions on manifolds with boundary. Advances in Applied Mathematics.

Konishi, S. and Fujita, H. (1994). A conveyance system using air flow based on the concept of distributed micro motion systems. Journal of Microelectromechanical Systems, 3(2):54-58.

Liu, W. and Will, P. (1995). Parts manipulation on an intelligent motion surface. In IEEE/RSJ Int. Workshop on Intelligent Robots 6 Systems (IROS), Pittsburgh, PA.

Luntz, J. E., Messner, W., and Choset, H. (1997). Parcel manipulation and dynamics with a distributed actuator array: The virtual vehicle. In Proc. IEEE Int. Conf. on Robotics and Automation (ICRA), pages 1541-1546, Albuquerque, New Mexico.

Lynch, K. (1996). Nonprehensile Robotic Manipulation. PhD thesis, The Robotics Institute, Carnegie Mellon University, Pittsburgh, PA.

McGeer, T. (1990). Passive dynamic walking. Int. Journal of Robotics Research.

Meriam, J. L. and Kraige, L. G. (1997). Engineering Mechanics - Statics. John Wiley and Sons, 4 edition.

Pister, K. S. J., Fearing, R., and Howe, R. (1990). A planar air levitated electrostatic actuator system. In Proc. IEEE Workshop on Micro Electro Mechanical Systems (MEMS), pages 67-71, Napa Valley, California.

Raibert, M. H., Hodgins, J. K., Playter, R. R., and Ringrose, R. P. (1993). Animation of legged maneuvers: jumps, somersaults, and gait transitions. Journal of the Robotics Society of Japan, 11(3):333-341.

Reif, J. and Wang, H. (1995). Social potential fields: A distributed behavioral control for autonoomous robots. In Goldberg, K., Halperin, D., Latombe, J.-C., and Wilson, R., editors, Algorithmic Foundations 
of Robotics (WAFR), pages 431-459. A. K. Peters, Ltd, Wellesley, MA.

Reznik, D. and Canny, J. F. (1998). Universal part manipulation in the plane with a single horizontally vibrating plate. In Agarwal, P. K., Kavraki, L., and Mason, M., editors, Robotics: The Algorithmic Perspective. A. K. Peters, Ltd, Wellesley, MA.

Rimon, E. and Koditschek, D. (1992). Exact robot navigation using artificial potential functions. IEEE Transactions on Robotics and Automation, 8(5).

Sandler, B.-Z. (1991). Robotics: Designing the Mechanisms for Automated Machinery. Prentice Hall.

Wiegley, J., Goldberg, K., Peshkin, M., and Brokowski, M. (1996). A complete algorithm for designing passive fences to orient parts. In Proc. IEEE Int. Conf. on Robotics and Automation (ICRA), pages 1133-1139, Minneapolis, MN. 